\title{
Comparison of diametric and volumetric changes in Stanford type $B$ aortic dissection patients in assessing aortic remodeling post-stent graft treatment
}

\author{
Wan Naimah Wan Ab Naim¹, Zhonghua Sun ${ }^{2}$, Yih Miin Liew ${ }^{3}$, Bee Ting Chan ${ }^{4}$, Shirley Jansen ${ }^{5,6,7}$, \\ Jing Lei ${ }^{8}$, Poo Balan Ganesan ${ }^{9}$, Shahrul Amry Hashim ${ }^{10}$, Ganiga Srinivasaiah Sridhar ${ }^{11}$, Einly Lim ${ }^{3}$ \\ ${ }^{1}$ Faculty of Mechanical and Automotive Engineering Technology, University Malaysia Pahang, 26600, Pekan, Pahang, Malaysia; ${ }^{2}$ Discipline of \\ Medical Radiation Science, Curtin University, Perth 6845, Australia; ${ }^{3}$ Department of Biomedical Engineering, Faculty of Engineering, University of \\ Malaya, 50603 Kuala Lumpur, Malaysia; ${ }^{4}$ Department of Mechanical, Materials and Manufacturing, Faculty of Science and Engineering, University \\ of Nottingham Malaysia, 43500 Semenyih, Selangor, Malaysia; ${ }^{5}$ Department of Vascular Surgery, Sir Charles Gairdner Hospital, Nedlands, Perth \\ WA 6009, Australia; ${ }^{6}$ Curtin Medical School, Curtin University, Perth 6845, Australia; ${ }^{7}$ University of Western Australia, Crawley WA 6009, \\ Australia; ${ }^{8}$ Department of Medical Imaging, First Affiliated Hospital of Kunming Medical University, Kunming, China; ${ }^{9}$ Department of Mechanical \\ Engineering, Faculty of Engineering, University of Malaya, 50603 Kuala Lumpur, Malaysia; ${ }^{10}$ Department of Surgery, Faculty of Medicine, \\ University of Malaya, 50603 Kuala Lumpur, Malaysia; ${ }^{11}$ Department of Medicine, Faculty of Medicine, University of Malaya, 50603 Kuala Lumpur, \\ Malaysia
}

Correspondence to: Einly Lim. Department of Biomedical Engineering, Faculty of Engineering, University of Malaya, 50603 Kuala Lumpur, Malaysia. Email: einly_lim@um.edu.my; Zhonghua Sun. Discipline of Medical Radiation Science, Curtin Medical School, Curtin University, Perth 6845, Australia. Email: z.sun@curtin.edu.au.

Background: The study aims to analyze the correlation between the maximal diameter (both axial and orthogonal) and volume changes in the true (TL) and false lumens (FL) after stent-grafting for Stanford type B aortic dissection.

Method: Computed tomography angiography was performed on 13 type B aortic dissection patients before and after procedure, and at 6 and 12 months follow-up. The lumens were divided into three regions: the stented area (Region 1), distal to the stent graft to the celiac artery (Region 2), and between the celiac artery and the iliac bifurcation (Region 3). Changes in aortic morphology were quantified by the increase or decrease of diametric and volumetric percentages from baseline measurements.

Results: At Region 1, the TL diameter and volume increased (pre-treatment: volume $=51.4 \pm 41.9 \mathrm{~mL}$, maximal axial diameter $=22.4 \pm 6.8 \mathrm{~mm}$, maximal orthogonal diameter $=21.6 \pm 7.2 \mathrm{~mm}$; follow-up: volume $=130.7 \pm 69.2 \mathrm{~mL}$, maximal axial diameter $=40.1 \pm 8.1 \mathrm{~mm}$, maximal orthogonal diameter $=31.9+2.6 \mathrm{~mm}$, $\mathrm{P}<0.05$ for all comparisons), while $\mathrm{FL}$ decreased (pre-treatment: volume $=129.6 \pm 150.5 \mathrm{~mL}$; maximal axial diameter $=43.0 \pm 15.8 \mathrm{~mm}$; maximal orthogonal diameter $=28.3 \pm 12.6 \mathrm{~mm}$; follow-up: volume $=66.6 \pm 95.0 \mathrm{~mL}$, maximal axial diameter $=24.5 \pm 19.9 \mathrm{~mm}$, maximal orthogonal diameter $=16.9 \pm 13.7, \mathrm{P}<0.05$ for all comparisons). Due to the uniformity in size throughout the vessel, high concordance was observed between diametric and volumetric measurements in the stented region with $93 \%$ and $92 \%$ between maximal axial diameter and volume for the true/false lumens, and $90 \%$ and $92 \%$ between maximal orthogonal diameter and volume for the true/false lumens. Large discrepancies were observed between the different measurement methods at regions distal to the stent graft, with up to $46 \%$ differences between maximal orthogonal diameter and volume.

Conclusions: Volume measurement was shown to be a much more sensitive indicator in identifying lumen

^ ORCID: 0000-0002-7538-4761. 
expansion/shrinkage at the distal stented region.

Keywords: Aortic dissection; maximal diameter; volume; aortic remodeling; computed tomography angiography

Submitted Jul 01, 2020. Accepted for publication Dec 10, 2020.

doi: $10.21037 /$ qims-20-814

View this article at: http://dx.doi.org/10.21037/qims-20-814

\section{Introduction}

Aortic stent-grafting procedure is less invasive compared with open surgery for type B aortic dissection (AD) patients, and has been shown to produce good clinical outcomes with low mortality rates (1). However, as the long-term results remain unknown, continuous assessment of the entire aorta after the procedure is crucial to detect acute or long-term complications (1). Currently, there is no best method to measure aortic changes after the stentgrafting repair (2). Although most studies have reported the application of maximal aortic diameters (1-9), several groups have also proposed using volumetric measurements as the most accurate way of monitoring aortic remodeling or ongoing expansion (10-16). Due to this heterogeneity in methods used, it is difficult to compare the results in the literature $(4,15)$. Moreover, the $\mathrm{AD}$ has highly irregular luminal shape, thus its measurement is complicated and may subject to large variability. Standardized quantification of the change in aortic dimension before and after the treatment along its whole length is crucial in determining which patients require further intervention in the presence of ongoing lumen expansion.

Thus, this study aims to examine the changes of the maximal axial as well as orthogonal diameters in different regions of the true lumen (TL) and false lumen (FL) to determine if they are reliable measures in assessing aortic remodeling of $\mathrm{AD}$ patients after undergoing stent-grafting. Concordance analysis is later performed for the percentage of measurement changes between maximal diameters (axial and orthogonal) and volume to ascertain if diametric measurements are sufficient in reflecting volumetric changes in the TL and FL after the repair.

\section{Methods}

\section{Image acquisition and measurements}

For this retrospective, single-center study, 13 extensive Stanford B AD patients who underwent computed tomography angiography (CTA) before and after the treatment at a major public hospital in Perth, Western Australia from 2004 to 2015, were selected. Patient inclusion criteria were as follows: Stanford type B AD, treated with endovascular stent grafts and underwent routine CTA follow-ups. Out of 13 patients, 11 patients were treated for acute while the remaining two patients were treated for chronic AD. The follow-up durations varied depending on the patients' conditions (minimum 6 months and maximum 72 months). To ensure consistency among patients, only data from the 6 and 12 months followup period after the stent-grafting procedures were selected. Thirty datasets from 13 patients were processed, with nine patients having two datasets (either post-treatment and 6 months, or post-treatment and 12 months) and four patients (Patients 1, 2, 5 and 8) having three datasets (posttreatment, 6 and 12 months). The study was approved by the institutional review board and the patients' identities were de-identified.

The patients' CTA images were transferred to the ScanIP software (Simpleware, Ltd, Exeter, UK), whereby the region of interest (ROI), including the ascending aorta (starting at the slices distal to the coronary artery origins), aortic arch, TL and FL of the descending thoracic aorta and abdominal aorta (ending at the aortic bifurcation), were identified and segmented on each axial slice. The segmented ROI were analysed using the MATLAB software (version r2012a, The MathworksInc, Natick, MA).

Axial diameters were calculated for the TL and FL regions from the start of the descending aorta (distal to the left subclavian artery) to the bifurcation (Figure 1A). At each axial plane (Figure 1B), the centroids of both the TL and FL were calculated (Figure 1C). The axial diameter of the TL and FL were measured along the line connecting the two centroids as shown in Figure 1D. In the nondissected aorta, diameter was measured perpendicular to the maximum elliptic diameter (3). Multiplanar reconstructions were also performed to obtain planes perpendicular to the central luminal line, and the orthogonal diameters were then calculated on these orthogonal planes using the same method described above for the axial diameter (as illustrated 


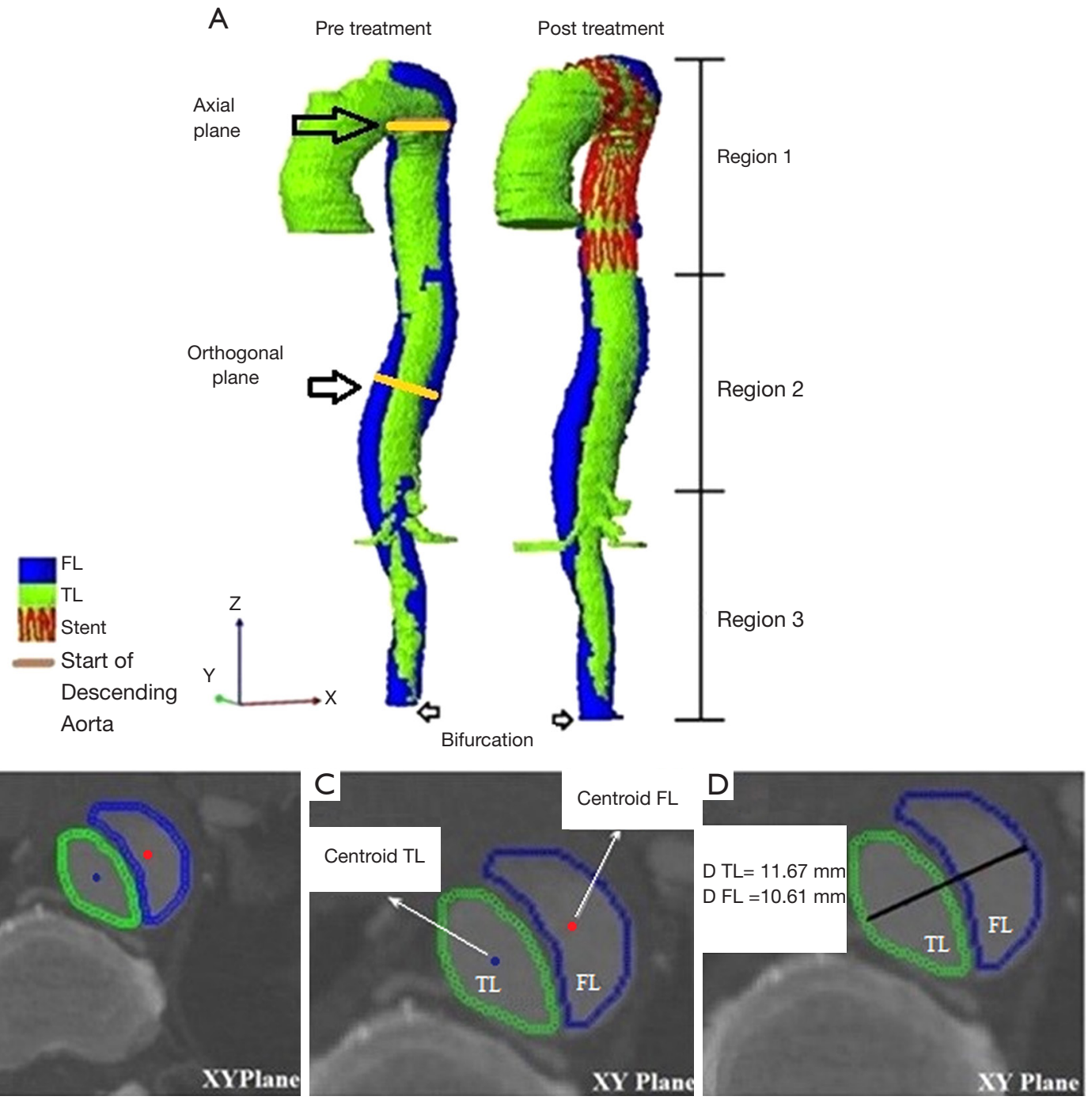

Figure 1 Visualisation workflow: (A) 3D reconstructed geometry of segmented AD aorta (TL and FL) during pre-treatment and posttreatment, (B) axial plane (X-Y Plane), (C) centroid points at both TL and FL, and (D) diameter of TL and FL. AD, aortic dissection; FL, false lumen; TL, true lumen.

in Figure 1D).

The TL and FL volumes were calculated by multiplying the corresponding area of ROI at each axial slice with the slice spacing, to estimate the ROI volume, and summing the corresponding ROI volume across all the slices. The algorithms for calculating the TL and FL volumes were also programmed using the MATLAB software. The average computational time was recorded.

In this study, we divided the aorta into three sections of interest (Figure 1A), i.e., the stented region (Region 1), the aortic region extending from the distal end of the stent graft to the origin of the celiac artery (Region 2-remaining thoracic aorta), and the region between the origin of the celiac artery and the bifurcation (Region 3-abdominal aorta). In order to map the stented region from the postoperative scans to the pre-operative scan, the start of Region 1 was defined as the image slice corresponding to the left subclavian artery for all time points, as this is the nearest landmark to the most common proximal landing zone of the stent graft. The image slice corresponding to the distal end of the stent in the post-operative slices was then identified and defined as the end of Region 1. The calculated length of Region 1 from the post-operative scan was used to identify the image slice corresponding to the 

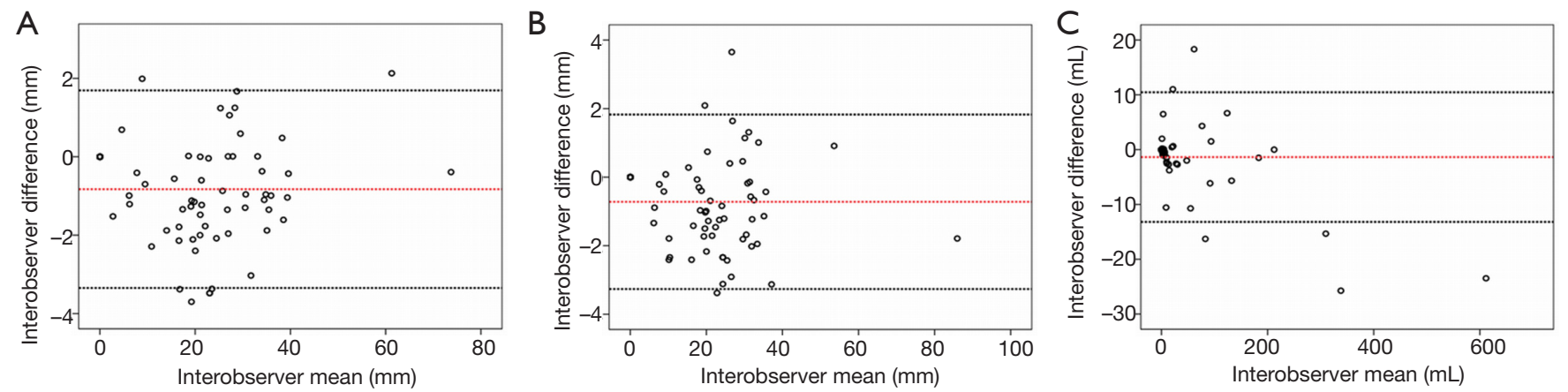

Figure 2 The Bland-Altman plots for (A) maximal axial diameter; (B) maximal orthogonal diameter and (C) volume.

end of Region 1 in the pre-operative scan. The TL and FL volumes for these three regions were measured separately. Apart from volume measurements, the maximum axial and orthogonal diameters of the $\mathrm{TL}$ and $\mathrm{FL}$ at each region were also determined by selecting the largest diameter measurement among all the slices/planes from each region (3).

\section{Interobserver variability analysis}

In order to assess interobserver variability, we have randomly selected nine datasets from 13 patients (total 30 datasets) covering pre-treatment, post-treatment and follow-up images. For each dataset, two interobservers were requested to perform segmentation of the aorta independently. There are a total of 1,359 slices segmented for nine datasets. From each dataset, seven to eight measurements of axial diameter, orthogonal diameter and volume from the TL and FL regions of each of the dataset were automatically generated by the in-house coding, yielding 70 measurements per parameter for Bland-Altman analysis (Figure 2). This analysis provided an understanding of the total bias (i.e., mean signed difference between observers) and limits of agreement (i.e., 95\% confidence interval or $1.96 \times$ standard deviation of the signed difference from the mean) among the observers. The Bland-Altman analysis was performed using SPSS version 22.0 (IBM SPSS Statistics, Armonk, NY, USA).

\section{Assessment of aortic remodeling}

The primary study endpoint is the percentage changes in maximal axial/orthogonal diameters and volume of the patients from baseline to subsequent follow-ups, which characterize the degree of aortic remodeling in these patients:

$$
\begin{aligned}
& \% \text { of change }=(\text { post treatment volume }- \\
&\text { pretreatment volume }) / \text { pretreatment } \times 100
\end{aligned}
$$

Luminal changes were identified based on a $10 \%$ cutoff point, as proposed by previously reported studies $(10,15)$, in which an increase in the luminal maximal axial diameter and volume was defined as $>10 \%$ increase from the baseline value; $\pm 10 \%$ from baseline defined a stable maximal axial diameter and volume, and a decrease in luminal maximal axial diameter and volume was defined as $>10 \%$ decline from the baseline value $(10,15)$.

Percentages of concordance and discordance between the maximal diameter and volume changes were then quantified. Percentage of concordance represents the percentage of segments with maximal diameter and volume changes showing the same trends (i.e., either both showing increments or both showing decrements based on the $10 \%$ cutoff point). In contrary, percentage of discordance represents the percentage of segments with volume and maximal diameter changes showing opposite trends (i.e., one showing an increment while another showing a decrement based on the $10 \%$ cutoff point). A $\mathrm{P}$ value of less than 0.05 is considered statistically significant difference.

\section{Results}

\section{Interobserver variability results}

The total bias of the maximal axial diameter measurement was $-0.83 \mathrm{~mm}$ with the variability in terms of $95 \%$ limits of agreement from -3.35 to $1.69 \mathrm{~mm}$, the total bias of the maximal orthogonal diameter measurement was 
Table 1 Morphology of the aorta after stent-grafting repair

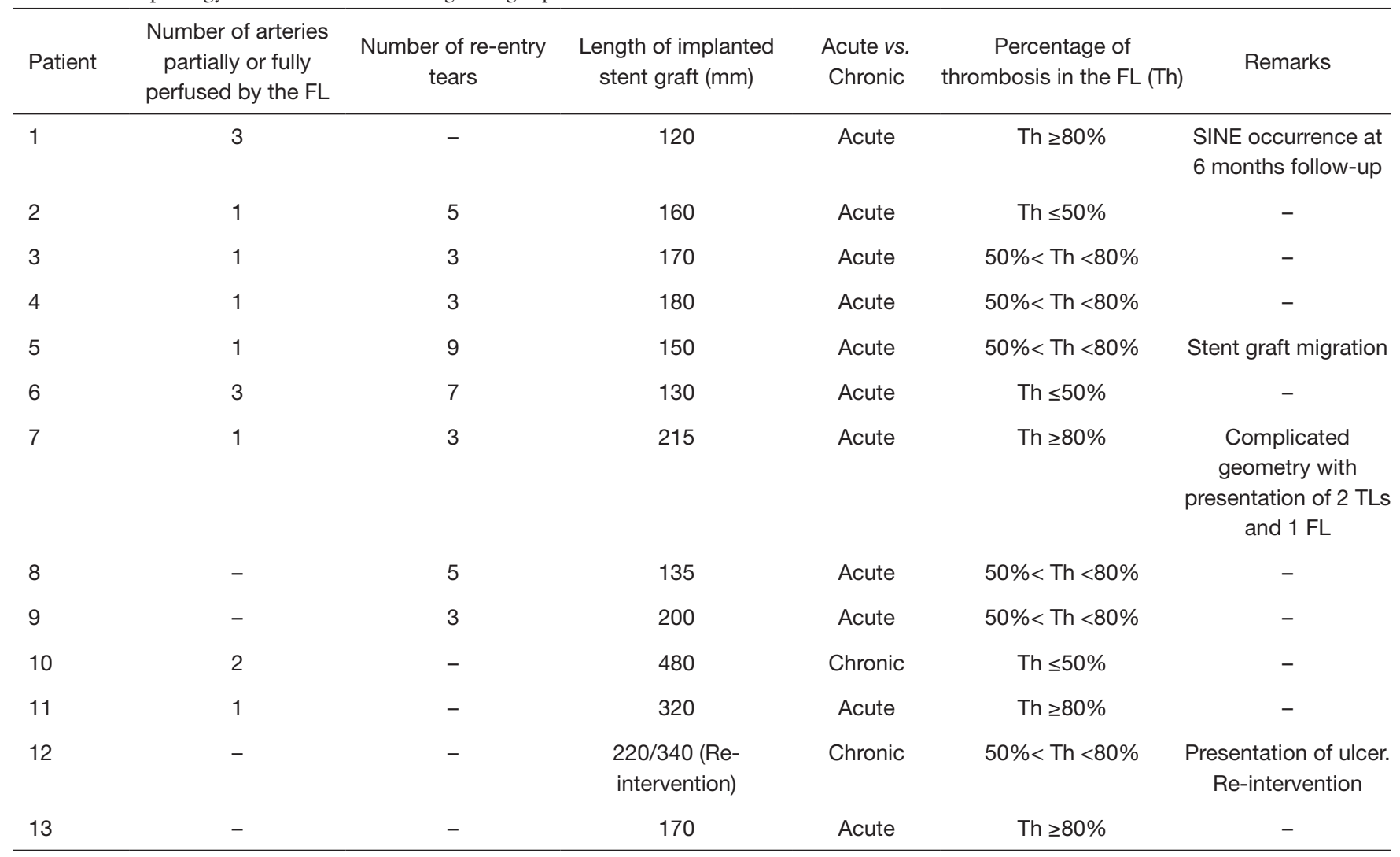

SINE, stent graft induced new entry tear; Th, thrombosis.

$-0.72 \mathrm{~mm}$ with $95 \%$ limits of agreement from -3.23 to $1.83 \mathrm{~mm}$. The total bias of the volumetric measurement was $-1.36 \mathrm{~mL}$ with $95 \%$ limits of agreement of -13.19 to $10.46 \mathrm{~mL}$. These results suggest that the measurements had sub-850-micron bias in estimating the aortic diameter which is in the ballpark of the image resolution (i.e., 600-850 microns).

\section{Mean TL/FL diametric and volumetric changes from pre- treatment to follow-ups in all patients}

Table 1 lists important morphological features of the aorta in all patients that may affect the degree of aortic remodeling, which include number of arteries partially or fully perfused by the FL, number of reentry tears, length of stent graft, as well as degree of FL thrombosis at the end of the followup period. Out of the 13 patients, four (Patients 1, 7, 11 and 13) achieved $>80 \%$ thrombosis, six (Patients 3, 4, 5, 8, 9, 12) achieved between $50-80 \%$ thrombosis, while the remaining patients (Patients 2, 6, 10) achieved $<50 \%$ thrombosis.

As shown in Figure 3A,B,C, the average maximal axial/ orthogonal diameters and volume of TL in 13 patients showed similar changes at each region. At Regions 1 and 2 , the TL diameter and volume increased throughout the study period.

Region 1 : mean volume $=51.4 \pm 41.9 \mathrm{~mL}$ during pretreatment and $130.7 \pm 69.2 \mathrm{~mL}$ at the follow-up; mean maximal axial diameter $=22.4 \pm 6.8 \mathrm{~mm}$ during pretreatment and $40.1 \pm 8.1 \mathrm{~mm}$ at the follow-up; mean maximal orthogonal diameter $=21.6 \pm 7.2 \mathrm{~mm}$ during pre-treatment and $31.9 \pm 2.6 \mathrm{~mm}$ at the follow-up. Pre-treatment and follow-up measurements showed significant differences ( $\mathrm{P}<0.05$ for all comparisons).

Region 2: mean volume $=17.4 \pm 6.8 \mathrm{~mL}$ during pretreatment and $39.5 \pm 13.3 \mathrm{~mL}$ at the follow-up; mean maximal axial diameter $=17.1 \pm 6.6 \mathrm{~mm}$ during pretreatment and $27.1 \pm 8.5 \mathrm{~mm}$ at the follow-up; mean maximal orthogonal diameter $=14.6 \pm 3.6 \mathrm{~mm}$ during pre-treatment 
A
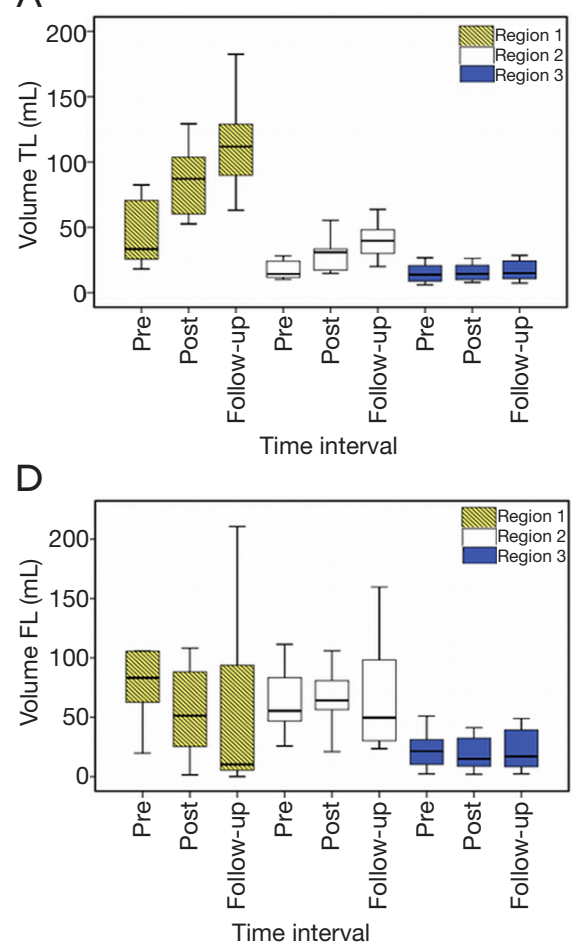

B

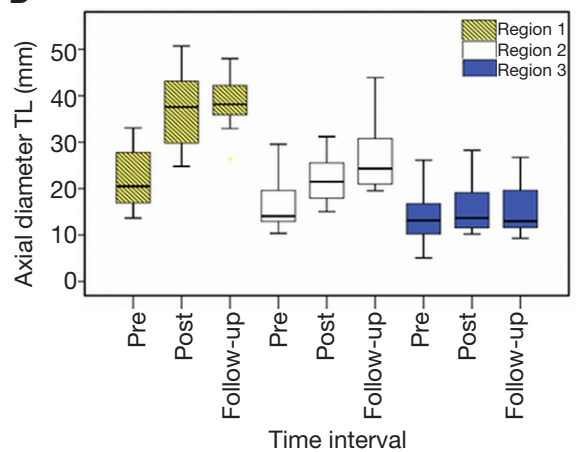

$\mathrm{E}$

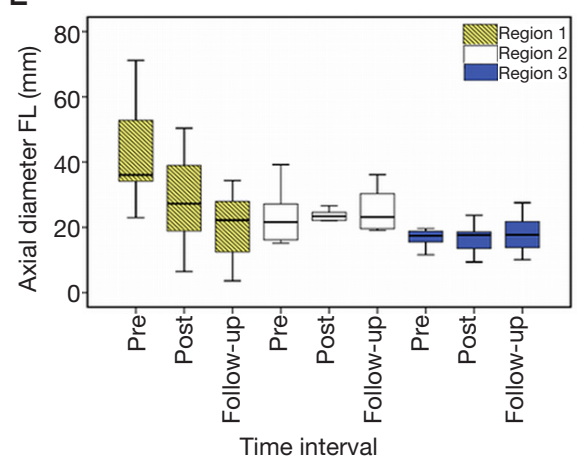

C

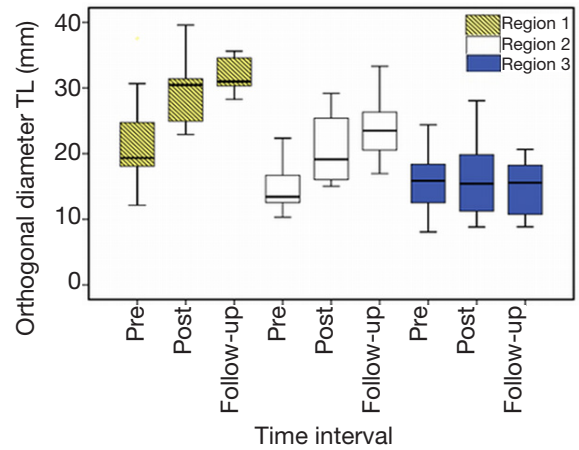

$\mathrm{F}$

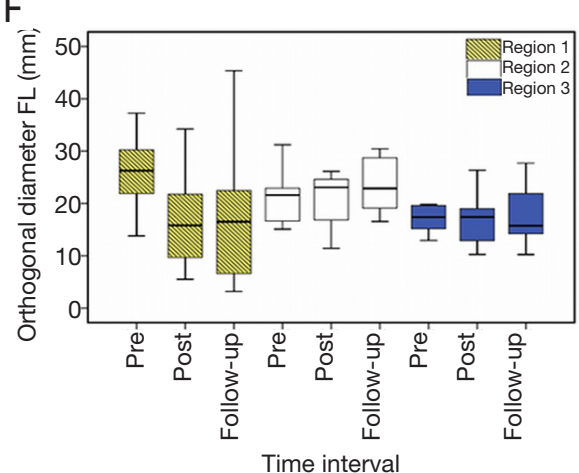

Figure 3 Boxplots showing the differences in TL volume (A), TL maximal axial diameter (B), TL maximal orthogonal diameter (C), FL volume (D), FL maximal axial diameter (E) and FL maximal orthogonal diameter (F) changes between pre-treatment, post-treatment and follow-up at Regions 1, 2 and 3. FL, false lumen; TL, true lumen.

and $23.2 \pm 5.1 \mathrm{~mm}$ at the follow-up).

Meanwhile, in Region 3, both TL maximal axial diameter and volume showed a slight increase from pre-stenting to the follow-up. On the other hand, maximal orthogonal diameter remained relatively constant throughout the study period.

Similar changes among the three different measurement techniques were also noticed in the FL regions, as shown in Figure 3D,E,F. As opposed to the TL, the Region 1 of the FL decreased from pre-stenting (mean volume $=129.6 \pm 150.5 \mathrm{~mL}$; mean maximal axial diameter $=43.0 \pm 15.8 \mathrm{~mm}$; mean maximal orthogonal diameter $=28.3 \pm 12.6 \mathrm{~mm}$ ) to the follow-up (mean volume $=66.6 \pm 95.0 \mathrm{~mL}$; mean maximal diameter $=24.5 \pm 19.9 \mathrm{~mm}$; mean maximal orthogonal diameter $=16.9 \pm 13.7 \mathrm{~mm}$ ). Pre-treatment and follow-up measurements showed significant differences ( $\mathrm{P}<0.05$ for all comparisons). On the other hand, there was no consistent trend in the mean maximal diameters and volume of the FL in Regions 2 and 3.

\section{Comparison between diametric and volumetric measurements in assessing aortic remodeling from pre- treatment to follow-ups}

Detailed changes in the percentage of maximal axial/ orthogonal diameter and volume from the baseline (pretreatment) of each patient were analysed at post-treatment, 6 and 12 months follow-ups for Regions 1, 2 and 3 of the TL and FL. Maximal axial/orthogonal diameter and volume during pre-treatment (i.e., baseline) as well as percentages of change in these parameters for each patient from the baseline to post-treatment and 6 months were shown in Tables 2 and 3 for the TL and FL, respectively. A positive percentages value indicated luminal expansion, whereas a negative percentages value showed luminal shrinkage. There were 153 comparisons each between the maximal diameters changes (axial and orthogonal), with volume changes (both TL and FL) for all regions throughout the follow-up period (Table 4). 
Table 2 Baseline measurement for the TL axial $\left(M a x D_{A x}\right)$ and orthogonal $\left(M a x D_{\mathrm{Or}_{r}}\right)$ diameter and volume, as well as their respective changes from baseline

\begin{tabular}{|c|c|c|c|c|c|c|c|c|c|}
\hline \multirow{2}{*}{ Patient } & \multicolumn{3}{|c|}{ Pre-treatment } & \multicolumn{3}{|c|}{ Post-treatment } & \multicolumn{3}{|c|}{6 months } \\
\hline & $\operatorname{MaxD}_{\mathrm{Or}}(\mathrm{mm})$ & $\operatorname{Max} D_{A x}(m m)$ & Vol (mL) & $\operatorname{Max} \mathrm{D}_{\mathrm{Or}}(\%)$ & $\operatorname{Max} D_{A x}(\%)$ & Vol (\%) & $\operatorname{Max} D_{\mathrm{Or}}(\%)$ & $\operatorname{Max} D_{A x}(\%)$ & Vol (\%) \\
\hline \multicolumn{10}{|c|}{ Region 1} \\
\hline 2 & 19.3 & 17.8 & 39.4 & 23.8 & 55.2 & 145.2 & 40.0 & 98.8 & 220.8 \\
\hline 3 & 18.2 & 16.5 & 32.0 & 36.9 & 72.7 & 94.1 & 79.7 & 122.2 & 180.6 \\
\hline 5 & 12.2 & 25.6 & 18.1 & 150.6 & 42.4 & 191.7 & 138.7 & 69.9 & 400.6 \\
\hline 6 & 16.9 & 22.7 & 20.4 & 36.1 & 73.8 & 194.2 & NA & NA & NA \\
\hline 7 & 18.8 & 16.9 & 29.7 & 22.5 & 76.2 & 181.0 & 70.7 & 122.2 & 235.9 \\
\hline 8 & 18.1 & 18.5 & 33.4 & 73.3 & 105.7 & 57.5 & 73.7 & 97.3 & 123.2 \\
\hline 12 & 36.1 & 37.5 & 82.5 & 5.7 & 5.3 & 122.9 & -4.4 & 5.9 & 130.6 \\
\hline 13 & 30.7 & 30.5 & 70.7 & 0.7 & 41.4 & 33.3 & 13.6 & 26.6 & 79.8 \\
\hline \multicolumn{10}{|c|}{ Region 2} \\
\hline 1 & 16.7 & 29.6 & 25.4 & 2.2 & 5.5 & 31.4 & 38.2 & 35.2 & 74.8 \\
\hline 2 & 13.2 & 14.1 & 14.3 & 44.9 & 52.5 & 220.3 & 43.9 & 60.5 & 171.3 \\
\hline 3 & 11.9 & 12.1 & 11.8 & 31.3 & 34.2 & 44.9 & 73.3 & 73.5 & 133.1 \\
\hline 4 & 12.6 & 12.9 & 12.6 & 80.8 & 70.6 & 146.0 & 66.3 & 62.9 & 138.9 \\
\hline 5 & 13.4 & 14.0 & 18.0 & 117.3 & 27.9 & -4.4 & 124.1 & 116.7 & 92.8 \\
\hline 1 & 17.9 & 21.9 & 22.7 & -3.6 & -0.1 & 12.2 & 0.6 & 1.5 & 14.4 \\
\hline 2 & 11.4 & 5.1 & 6.0 & 25.7 & 178.7 & 98.3 & 40.5 & 142.7 & 116.7 \\
\hline 3 & 13.9 & 10.6 & 8.6 & -9.2 & 24.3 & 14.0 & -15.2 & 15.0 & 22.1 \\
\hline 4 & 19.6 & 12.7 & 16.1 & -1.5 & -7.5 & -1.4 & -18.6 & 7.2 & 22.1 \\
\hline 5 & 13.6 & 13.5 & 16.0 & 5.8 & 4.7 & 1.9 & 0.4 & 5.4 & 5.0 \\
\hline 6 & 8.1 & 8.6 & 10.6 & 22.1 & 18.3 & 26.3 & NA & NA & NA \\
\hline 7 & 18.9 & 11.9 & 26.8 & 10.9 & -4.2 & -1.5 & -2.6 & -1.4 & 2.1 \\
\hline 8 & 15.5 & 15.8 & 18.7 & -42.9 & -35.0 & -46.2 & -41.9 & -25.3 & -27.7 \\
\hline 9 & 24.4 & 26.1 & 48.2 & 15.1 & 8.1 & 19.6 & 53.8 & 53.0 & 64.3 \\
\hline 10 & 9.2 & 9.8 & 8.7 & -0.5 & 29.0 & -9.5 & NA & NA & NA \\
\hline 11 & 16.6 & 16.7 & 8.9 & -1.3 & -1.6 & -4.7 & -8.4 & -1.3 & -0.2 \\
\hline 12 & 16.3 & 16.8 & 11.7 & 25.5 & 31.0 & 31.3 & 26.8 & 59.1 & 20.6 \\
\hline
\end{tabular}

NA, not available. 
Table 3 Baseline measurement for the FL axial $\left(\mathrm{Max} \mathrm{D}_{\mathrm{Ax}}\right)$ and orthogonal $\left(\mathrm{Max} \mathrm{D}_{\mathrm{Or}}\right)$ diameter and volume, as well as their respective changes from baseline

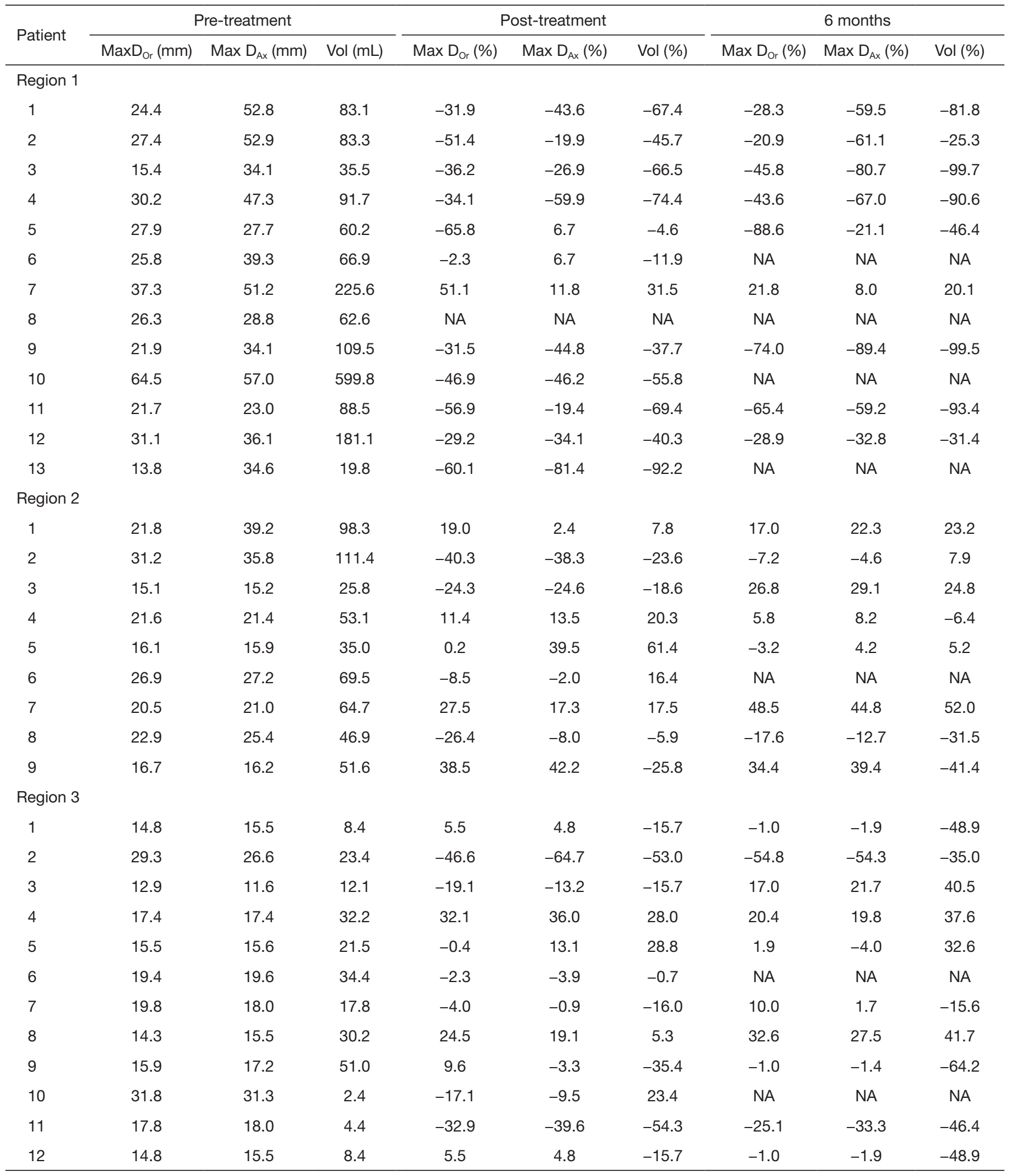

NA, not available. 
Table 4 The agreement between maximal diameters (axial and orthogonal) and volume changes at each region of the TL and FL

\begin{tabular}{|c|c|c|c|c|}
\hline & \multicolumn{2}{|c|}{ Maximal axial diameter vs. volume } & \multicolumn{2}{|c|}{ Maximal orthogonal diameter vs. volume } \\
\hline & $\%$ of concordance & $\%$ of discordance & $\%$ of concordance & $\%$ of discordance \\
\hline \multicolumn{5}{|l|}{ TL } \\
\hline Region 1 & $93 \%$ & $7 \%$ & $90 \%$ & $10 \%$ \\
\hline Region 2 & $82 \%$ & $18 \%$ & $91 \%$ & $9 \%$ \\
\hline Region 3 & $71 \%$ & $29 \%$ & $68 \%$ & $32 \%$ \\
\hline \multicolumn{5}{|l|}{$\mathrm{FL}$} \\
\hline Region 1 & $92 \%$ & $8 \%$ & $92 \%$ & $8 \%$ \\
\hline Region 2 & $73 \%$ & $27 \%$ & $59 \%$ & $41 \%$ \\
\hline Region 3 & $62 \%$ & $38 \%$ & $54 \%$ & $46 \%$ \\
\hline
\end{tabular}

\section{True lumen}

In Region 1 TL (Table 2), the maximal axial and orthogonal diameters as well as volume for all patients showed drastic increase from pre-treatment, except for Patients 12 and 13. Patient 12 showed stable maximal orthogonal and axial diameters at post-treatment and 6 months follow-up while Patient 13 showed stable maximal orthogonal diameter at post-treatment. $93 \%$ concordance was observed between maximal axial diameter and volume changes, while $90 \%$ concordance was found between maximal orthogonal diameter and volume changes (Table 4). In most of the cases, as compared to diametric measurements (either axial or orthogonal), the percentage changes from baseline to posttreatment and follow-ups were found to be much higher using the volumetric measurement.

Meanwhile, in Region $2 \mathrm{TL}$, maximal axial and orthogonal diameters as well as volume appeared to increase throughout the study period except for Patient 1 (posttreatment), Patient 5 (post-treatment) and Patient 9 (posttreatment and 6 months) (Table 2). Patient 1 showed an increment in the volume but with stable maximal diameters (both axial and orthogonal), while Patient 9 showed an increment in the volume and maximal orthogonal diameter but with a stable maximal axial diameter. Patient 5 , whose scan image was presented in Figure 4, showed an increase in maximal axial and orthogonal diameters, but had a stable volume (Table 2). These differences resulted in a discordance of $18 \%$ between maximal axial diameter and volume changes, and $9 \%$ between maximal orthogonal diameter and volume changes (Table 4).

Unlike Regions 1 and 2 TL, which demonstrated an increase in size for most patients, there was inconsistent trend in all the three measurement methods for Region 3 TL. Furthermore, several patients showed different trends (i.e., stable, increment or decrement) when assessed using the different measures (maximal axial, orthogonal diameter and volume). These differences represented 29\% of the discordance maximal axial diameter and volume changes, and $32 \%$ between maximal orthogonal diameter and volume changes (Table 4). The possible reason for the changes discrepancies in the Regions 2 and 3 in the TL was because of the presentation of stent graft induced new entry tear (SINE) i.e., in Patient 1 and the stent graft migration that resulted in localized expansion in the case of Patient 5 as shown in Figure 4 (Table 1).

\section{False lumen}

As opposed to Region $1 \mathrm{TL}$, Region $1 \mathrm{FL}$ in most patients (Table 3) showed drastic shrinkage for maximal axial/ orthogonal diameters and volume from the baseline. A decrement in size was captured by volume for Patient 6 at post-treatment (Figure 5), but not with maximal axial or orthogonal diameters (which showed stable diameter). Similarly, Patient 7 showed an increase in both maximal orthogonal diameter and volume at 6 months, but with a stable axial diameter. As a result, $92 \%$ concordance was found between maximal axial/orthogonal diameters and volume changes.

In contrary to Region $1 \mathrm{FL}$, a much higher discordance was observed between maximal axial/orthogonal diameters and volume changes (Tables 3 and 4) in Region 2 (27\% between maximal axial diameter and volume; $41 \%$ between maximal orthogonal diameter and volume) and Region 3 (38\% between maximal axial diameter and volume; 


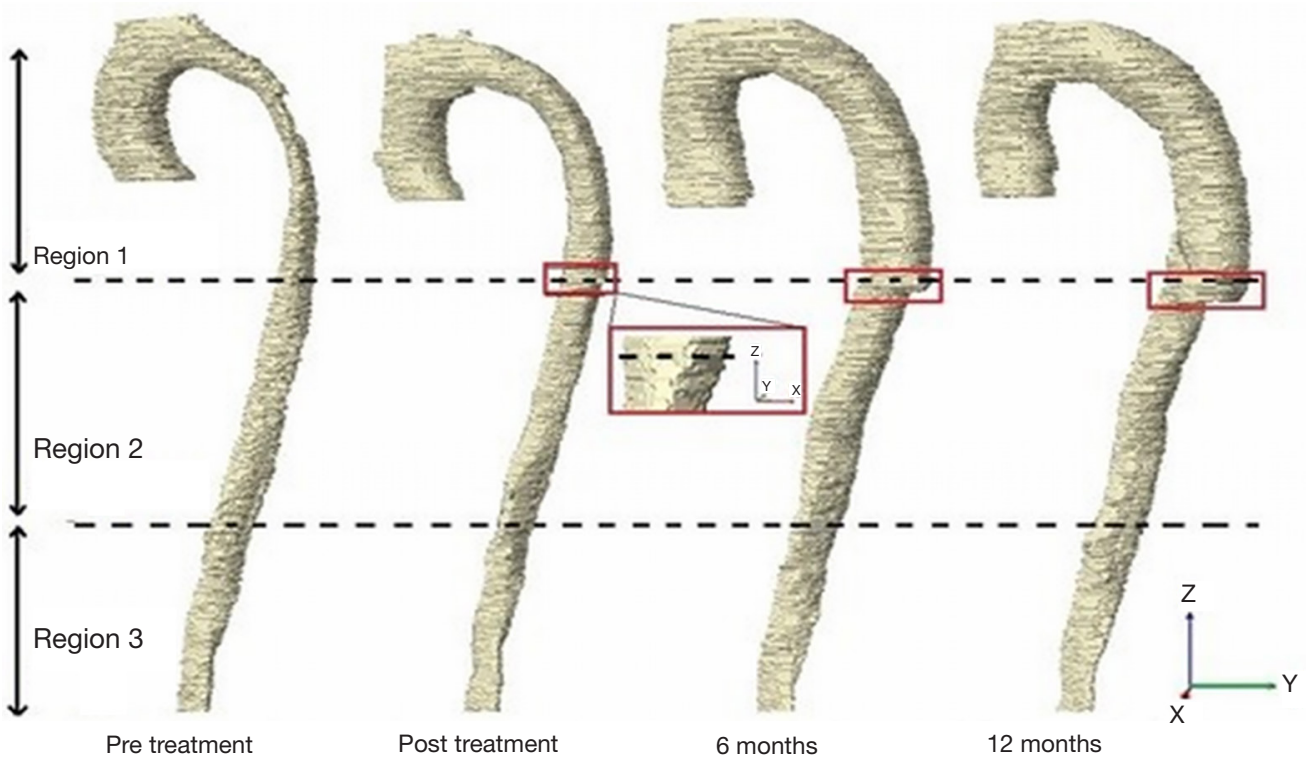

Figure 4 Localized expansion (red rectangular box) of the stent graft edge (Region 2 TL) during post treatment for Patient 5 , which increased at 6 and 12 months follow-up. TL, true lumen.

$46 \%$ between maximal orthogonal diameter and volume). Volumetric measurement was observed to be much more sensitive in detecting changes in the FL size in Regions 2 and 3. At post-treatment and 6 months, Patients 1, 7, 9 and 12 have stable maximal axial and orthogonal diameters but the volume was observed to decrease.

\section{Discussion}

This is the first study which compares three main measurement methods, i.e., maximal axial diameter, maximal orthogonal diameter and volume in assessing the TL and FL changes in AD patients after stent-grafting repair. Although the feasibility of using maximal axial diameter and volume changes to indicate blood vessel remodeling have been studied in the abdominal aortic aneurysms (AAAs) (17-19), the relationship between maximal diametric and volumetric changes in Type $\mathrm{B} A D$ after the stent insertion presents a major challenge as $\mathrm{AD}$ cases involve both the TL and FL, either of which can significantly change shape after treatment (10). Furthermore, the dissection usually extends over a long distance, with several major branches extending from the main vessels involved (20).

Most studies monitored aortic remodeling by measuring the whole vessel (from the top of the aortic arch to the lowest renal artery level) as a single unit (13). Measurement of the TL maximal diameter along the whole vessel without separation into different regions would neglect changes in areas other than the stented region. Separately capturing changes in dimensions distal to the stent graft is necessary, as studies have shown an expansion particularly in the FL section, after the repair $(4,10,15)$. Therefore, in this work, the vessel has been separated into three regions in order to distinguish the differences in change along the entire aorta beyond the left subclavian artery.

As expected, the findings showed that the whole Region 1 in the TL consistently increased in size after the stent insertion. Self-expandable stents implanted in our patients continuously exert radially outward expanding force on the vessel wall, leading to negative chronic recoil and TL expansion over time (21). An increase in volume in the stented region during follow-up has also been previously reported in other studies $(10,13)$. The uniformity in size observed throughout Region 1 from the proximal to the distal regions resulted in a high concordance between maximal diameters and volumes (Table 4). Based on this finding, we conclude that the maximal axial diameter measurements alone have potential to monitor changes in the stented TL region after stent grafting procedure.

On the other hand, the higher sensitivity of volume measurement in capturing lumen size changes after stent grafting becomes more apparent in regions distal to the 
Pre treatment
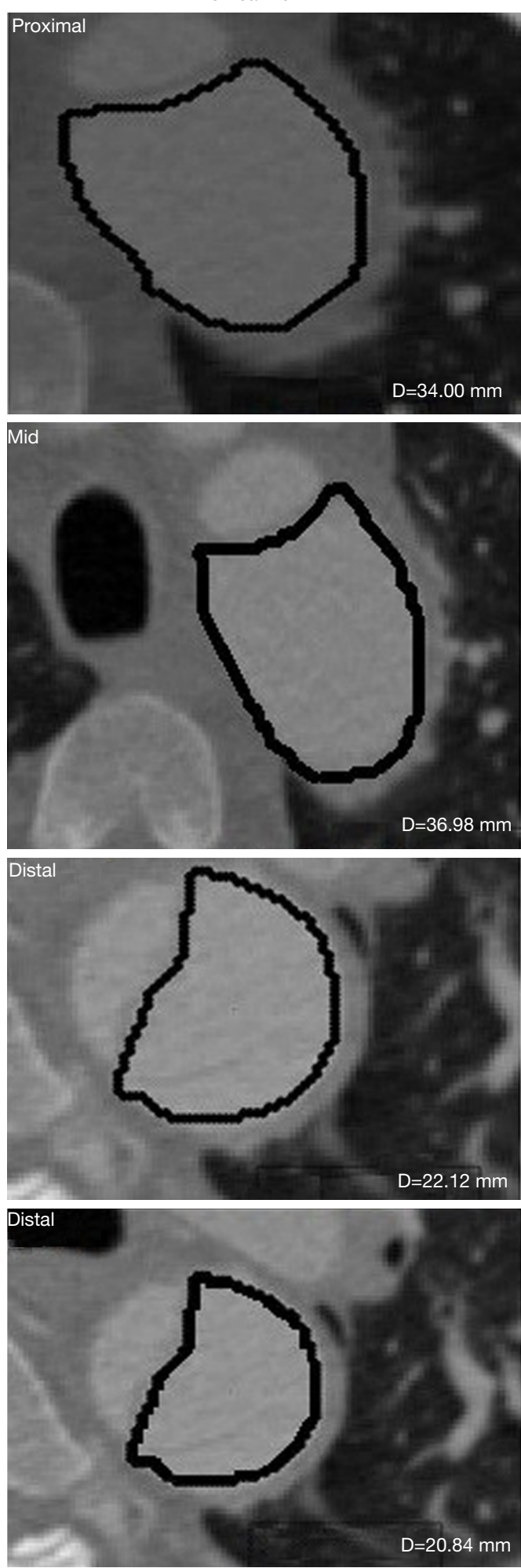

Post treatment
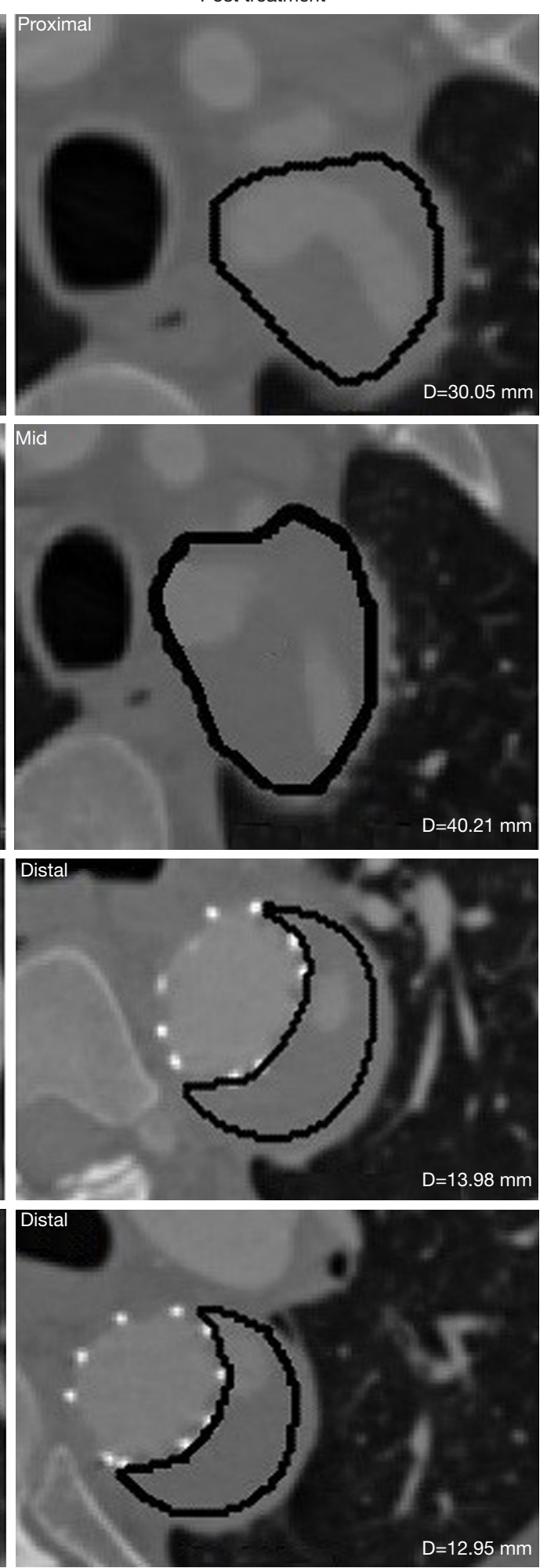

Figure 5 Selected axial slices from three different regions (proximal, mid and distal) along Region 1 of Patient 6 pre- and post-treatment. The solid contours represent the region of interest for FL, while D represents the calculated diameter. FL, false lumen. 
stent graft especially in the FL area (i.e., Regions 2 and 3). One third of the FL volume changes was not observed using either maximal axial or orthogonal diameter measurements in Regions 2 and 3 during post-treatment and follow-ups (Table 4). Due to the complex morphology of the aorta, maximal diameters may be located at different positions along the vessel depending on disease progression, and therefore determination of aortic remodeling based on these measurements may be prone to error. Along the extension of the aortic lumen, some parts may shrink while others may be dilated or remain stable (unchanged). For example, in several cases in our study, opposite trends were observed between maximal diametric and volumetric measurements. This situation occurred due to the inconsistency in lumen size changes along the region, such as the one happening in Patient 9 (Region $2 \mathrm{FL}$ at post-treatment), where the proximal part shrunk while other regions showed an increment in diameter during post-treatment. Consequently, it is difficult to assess changes along the lumen using diametric measurement alone. Volumetric measurement at different regions along the vessel (for example, above and below the stent region) is more sensitive to monitor morphological changes for both TL and FL after stent-grafting procedure, especially at region distal to the stent graft.

However, for cases involving localized luminal expansion, such as that frequently occurring distal to the stent graft edge, the maximal diametric measurement should be used together with the volumetric measurement to detect abrupt changes in lumen size at a particular location. For instance, localized expansion was observed distal to the stent graft edge during post-treatment in Patient 5 (Region $2 \mathrm{TL}$ ), most probably due to placement of the stent graft, as distal stent graft migration was noticed in this particular location during the 6 months of follow-up. Using diametric measurements, an increase in maximal axial/orthogonal diameters was detected at this region. This localized change in diameter did not cause a significant change (i.e., $>10 \%$ cutoff point) in the overall volumetric measurement.

The implantation of stent grafts in $\mathrm{AD}$ patients aims to not only expand the TL diameter, but also to reduce the FL size and flow in order to enable long-term aortic remodeling and prevent aneurysmal expansion. Therefore, it is crucial to have a sensitive measurement protocol which could accurately monitor the changes in the TL and FL sizes, especially at regions distal to the stent graft, as these regions have been reported to show unstable volumetric growth (aneurismal dilatation) after the post-interventional year (16) which requires timely reintervention. According to Czermak et al. (15), the FL distal to the stent graft appeared to be a weak region as the presence of stent grafts imposed high wall stress at this location. Furthermore, re-entry tears and arterial branches partially or fully supplied by the FL, normally present in the unstented FL region (i.e., Regions 2 and 3 in this study), and could cause continuous flow in the FL, which hinders the formation of FL thrombus in the FL. While complete thrombosis protects the patient from the ongoing risk of aneurysmal degeneration, patent or partial thrombosis could cause FL expansion over time. Based on our results, only four patients achieved near complete thrombosis, with a resultant reduction in $\mathrm{FL}$ volume from pre-treatment to follow-ups. The ability to precisely identify the lumen expansion had major implications, as misclassification could cause an underestimation of FL growth that might lead to increased risk of rupture. Of note, no consistent patterns were observed between acute $v s$. chronic AD patients as well as between patients with complete $v s$. incomplete FL thrombosis with regards to the comparison between maximal axial diameter and volume measurements.

Volume assessment is time-consuming as it requires additional processing, such as image segmentation and reconstruction $(17,19)$. Future work includes the development of an automated segmentation technique to save time. The automated extraction of TL and FL volumes using the MATLAB software took an average of 15 minutes.

One limitation of the present study is the inclusion of only a small number of patients. However, our analysis involved a total number of 153 comparisons between the maximal axial diameter and volume (as shown in Table 4), from different aortic regions for both TL and FL. Another limitation is the lack of the long-term followup in most of the patients. Further studies will need to address these limitations by including more patients with extended follow-up periods to monitor the dimensional and volumetric changes using this approach.

\section{Conclusions}

This study explores whether the maximal axial or orthogonal diameter measurement is sufficient in representing overall change in morphology in the adjacent regions after aortic stent grafting treatment for $\mathrm{AD}$. Our results showed that diameter measurement has the potential to indicate aortic remodeling at the stented region (Region 1), which showed consistent changes along the stent length. However, 
volumetric measurement was shown to be a much more sensitive indicator in assessing changes in the TL and FL sizes at regions distal to the stent graft and should therefore provide supportive information to reflect morphologic changes in both TL and FL at this region. As subsequent intervention is indicated in the presence of an expanding lumen to prevent or treat aneurysmal degeneration, and this is currently based on diameter measurement alone, the use of volumetric data may guide clinicians in deciding whether further intervention is warranted in the presence of an increase in diameter.

\section{Acknowledgments}

Funding: The research is supported by the Royal SocietyNewton Advanced Fellowship (NA170094/IF003-2018) and Impact-Oriented Interdisciplinary Research Grant (IIRG024B-2019). Wan Naimah Wan Ab Naim is the recipient of University Malaysia Pahang (UMP) PostDoctoral Fellowship in Research.

\section{Footnote}

Conflicts of Interest: All authors have completed the ICMJE uniform disclosure form (available at http://dx.doi. org/10.21037/qims-20-814). ZS serves as an unpaid associate editor of Quantitative Imaging in Medicine and Surgery. The other authors have no conflicts of interest to declare.

Ethical Statement: The study was approved by the institutional review board and the patients' identities were de-identified.

Open Access Statement: This is an Open Access article distributed in accordance with the Creative Commons Attribution-NonCommercial-NoDerivs 4.0 International License (CC BY-NC-ND 4.0), which permits the noncommercial replication and distribution of the article with the strict proviso that no changes or edits are made and the original work is properly cited (including links to both the formal publication through the relevant DOI and the license). See: https://creativecommons.org/licenses/by-nc-nd/4.0/.

\section{References}

1. Kusagawa H, Shimono T, Ishida M, Suzuki T, Yasuda F, Yuasa U, Onoda K, Yada I, Hirano T,Takeda K. Changes in false lumen after transluminal stent-graft placement in aortic dissections six years' experience. Circulation 2005;111:2951-7.

2. Conrad MF, Crawford RS, Kwolek CJ, Brewster DC, Brady TJ,Cambria RP. Aortic remodeling after endovascular repair of acute complicated type B aortic dissection. J Vasc Surg 2009;50:510-7.

3. Manning BJ, Dias N, Manno M, Ohrlander T, Malina M, Sonesson B, Resch T,Ivancev K. Endovascular treatment of acute complicated type B dissection: morphological changes at midterm follow-up. J Endovasc Ther 2009;16:466-74.

4. Schoder M, Czerny M, Cejna M, Rand T, Stadler A, Sodeck GH, Gottardi R, Loewe C,Lammer J. Endovascular repair of acute type B aortic dissection: longterm follow-up of true and false lumen diameter changes. Ann Thorac Surg 2007;83:1059-66.

5. Evangelista A, Salas A, Ribera A, Ferreira-González I, Cuellar H, Pineda V, González-Alujas T, Bijnens B, Permanyer-Miralda G,Garcia-Dorado D. Long-term outcome of aortic dissection with patent false lumen predictive role of entry tear size and location. Circulation 2012;125:3133-41.

6. Rodriguez JA, Olsen DM, Lucas L, Wheatley G, Ramaiah V,Diethrich EB. Aortic remodeling after endografting of thoracoabdominal aortic dissection. J Vasc Surg 2008;47:1188-94.

7. Lombardi JV, Cambria RP, Nienaber CA, Chiesa R, Mossop P, Haulon S, Zhou Q,Jia F. Aortic remodeling after endovascular treatment of complicated type B aortic dissection with the use of a composite device design. J Vasc Surg 2014;59:1544-54.

8. Kamman AV, van Herwaarden JA, Orrico M, Nauta FJ, Heijmen RH, Moll FL,Trimarchi S. Standardized protocol to analyze computed tomography imaging of type B aortic dissections. J Endovasc Ther 2016;23:472-82.

9. Mani K, Clough R, Lyons O, Bell R, Carrell T, Zayed $\mathrm{H}$, Waltham M,Taylor P. Predictors of outcome after endovascular repair for chronic type B dissection. Eur J Vasc Endovasc Surg 2012;43:386-91.

10. Steingruber IE, Chemelli A, Glodny B, Hugl B, Bonatti J, Hiemetzbeger R, Jaschke W,Czermak BV. Endovascular repair of acute type $\mathrm{B}$ aortic dissection: midterm results. J Endovasc Ther 2008;15:150-60.

11. Huptas S, Mehta RH, KÜhl H, Tsagakis K, Reinsch N, Kahlert P, Jakob HG,Eggebrecht H. Aortic remodeling in type B aortic dissection: effects of endovascular stentgraft repair and medical treatment on true and false lumen 
volumes. J Endovasc Ther 2009;16:28-38.

12. Stanley GA, Murphy EH, Knowles M, Ilves M, Jessen ME, Dimaio JM, Modrall JG,Arko FR. Volumetric analysis of type B aortic dissections treated with thoracic endovascular aortic repair. J Vasc Surg 2011;54:985-92.

13. Qing KX, Yiu WK, Cheng SW. A morphologic study of chronic type B aortic dissections and aneurysms after thoracic endovascular stent grafting. J Vasc Surg 2012;55:1268-75; discussion 1275-6.

14. Kim KM, Donayre CE, Reynolds TS, Kopchok GE, Walot I, Chauvapun JP, White RA. Aortic remodeling, volumetric analysis, and clinical outcomes of endoluminal exclusion of acute complicated type B thoracic aortic dissections. J Vasc Surg 2011;54:316-24.

15. Czermak BV, Mallouhi A, Perkmann R, Steingruber IE, Waldenberger P, Neuhauser B, Fraedrich G, Jung T,Jaschke WR. Serial CT volume and thrombus length measurements after endovascular repair of Stanford type B aortic dissection. J Endovasc Ther 2004;11:1-12.

16. Kim TH, Ko YG, Woo Kwon S, Choi D, Yun Lee D, Shim WH, Su Hyon M. Large false lumen area is a predictor of failed false lumen volume reduction after stent-graft repair in type B aortic dissection. J Endovasc Ther 2014;21:697-706.

17. Bargellini I, Cioni R, Petruzzi P, Pratali A, Napoli V, Vignali C, Ferrari M,Bartolozzi C. Endovascular repair of abdominal aortic aneurysms: analysis of aneurysm volumetric changes at mid-term follow-up. Cardiovasc Intervent Radiol 2005;28:426-33.

18. Parr A, Jayaratne C, Buttner P,Golledge J. Comparison of volume and diameter measurement in assessing small abdominal aortic aneurysm expansion examined using computed tomographic angiography. Eur J Radiol 2011;79:42-7.

19. Wever JJ, Blankensteijn J, Mali WTM, Eikelboom B. Maximal aneurysm diameter follow-up is inadequate after endovascular abdominal aortic aneurysm repair. Eur J Vasc Endovasc Surg 2000;20:177-82.

20. Litmanovich D, Bankier AA, Cantin L, Raptopoulos V, Boiselle PM. CT and MRI in diseases of the aorta. AJR Am J Roentgenol 2009;193:928-40.

21. Duerig T,Wholey M. A comparison of balloon-and selfexpanding stents. Minim Invasive Ther Allied Technol 2002;11:173-8.

Cite this article as: Wan Ab Naim WN, Sun Z, Liew YM, Chan BT, Jansen S, Lei J, Ganesan PB, Hashim SA, Sridhar GS, Lim E. Comparison of diametric and volumetric changes in Stanford type B aortic dissection patients in assessing aortic remodeling post-stent graft treatment. Quant Imaging Med Surg 2021;11(5):1723-1736. doi: 10.21037/qims-20-814 